\title{
Comorbidity prevalence among cancer patients: a population-based cohort study of four cancers
}

Helen Fowler ${ }^{1 *}$ D , Aurelien Belot ${ }^{1}$, Libby Ellis ${ }^{1}$, Camille Maringe ${ }^{1}$, Miguel Angel Luque-Fernandez ${ }^{2,3}$, Edmund Njeru Njagi ${ }^{1}$, Neal Navani ${ }^{4,5}$, Diana Sarfati ${ }^{6}$ and Bernard Rachet ${ }^{1}$

\begin{abstract}
Background: The presence of comorbidity affects the care of cancer patients, many of whom are living with multiple comorbidities. The prevalence of cancer comorbidity, beyond summary metrics, is not well known. This study aims to estimate the prevalence of comorbid conditions among cancer patients in England, and describe the association between cancer comorbidity and socio-economic position, using population-based electronic health records.

Methods: We linked England cancer registry records of patients diagnosed with cancer of the colon, rectum, lung or Hodgkin lymphoma between 2009 and 2013, with hospital admissions records. A comorbidity was any one of fourteen specific conditions, diagnosed during hospital admission up to 6 years prior to cancer diagnosis. We calculated the crude and age-sex adjusted prevalence of each condition, the frequency of multiple comorbidity combinations, and used logistic regression and multinomial logistic regression to estimate the adjusted odds of having each condition and the probability of having each condition as a single or one of multiple comorbidities, respectively, by cancer type.

Results: Comorbidity was most prevalent in patients with lung cancer and least prevalent in Hodgkin lymphoma patients. Up to two-thirds of patients within each of the four cancer patient cohorts we studied had at least one comorbidity, and around half of the comorbid patients had multiple comorbidities. Our study highlighted common comorbid conditions among the cancer patient cohorts. In all four cohorts, the odds of having a comorbidity and the probability of multiple comorbidity were consistently highest in the most deprived cancer patients.

Conclusions: Cancer healthcare guidelines may need to consider prominent comorbid conditions, particularly to benefit the prognosis of the most deprived patients who carry the greater burden of comorbidity. Insight into patterns of cancer comorbidity may inform further research into the influence of specific comorbidities on socio-economic inequalities in receipt of cancer treatment and in short-term mortality.
\end{abstract}

Keywords: Cancer, Comorbidity, Multimorbidity, Deprivation, Prevalence, England, Epidemiology

\footnotetext{
* Correspondence: Helen.Fowler@lshtm.ac.uk

${ }^{1}$ Cancer Survival Group, Department of Non-Communicable Disease

Epidemiology, London School of Hygiene \& Tropical Medicine, Keppel Street,

London WC1E 7HT, UK

Full list of author information is available at the end of the article
}

(c) The Author(s). 2020 Open Access This article is distributed under the terms of the Creative Commons Attribution 4.0 International License (http://creativecommons.org/licenses/by/4.0/), which permits unrestricted use, distribution, and reproduction in any medium, provided you give appropriate credit to the original author(s) and the source, provide a link to the Creative Commons license, and indicate if changes were made. The Creative Commons Public Domain Dedication waiver (http://creativecommons.org/publicdomain/zero/1.0/) applies to the data made available in this article, unless otherwise stated. 


\section{Background}

Comorbidity refers to the existence of a long-term health condition in the presence of a primary disease of interest [1]. Having one or more comorbidities may influence the patient's prognosis for a primary disease such as cancer. Comorbidity may influence the timing of cancer diagnosis, in either a positive or a negative way. For example, the symptoms of comorbidity may drive a patient to seek medical care sooner, potentially leading to an earlier diagnosis. Alternatively, cancer symptoms may be mistakenly considered as symptoms of a pre-existing health condition, and could delay diagnosis [2-4]. Following diagnosis, the presence of comorbidity may also influence timing, receipt, or outcome of treatment, with clear evidence that those with comorbidity are less likely to receive curative treatment than those without, despite increasing evidence that many patients with comorbidity benefit from such treatment [3]. Although the presence of multiple co-existent health conditions is commonplace, the guidelines, funding and structures of primary care may not support the care of more patients with multiple conditions [5], and care in secondary and tertiary centres is typically highly siloed [3].

Methods used in the scientific literature to describe, measure and quantify the status of comorbidity as an explanatory factor in adverse disease outcomes are varied. Many summarised metrics of comorbidity have been proposed, providing an overall picture of a patient's comorbidity status, some specific to a primary disease while others are more general. For example, a widely used metric of comorbidity in epidemiological studies is the Charlson Comorbidity Index (CCI) [6], which weights 19 long-term health conditions according to their relative risk of one-year mortality, to produce an overall index score.

In this study, we firstly aimed to examine the prevalence of comorbid conditions in cancer patients using English population-based electronic health records of patients diagnosed with cancer of the colon, rectum, or lung or with Hodgkin lymphoma (HL). An association between comorbidity (not specific to any primary disease of interest) and socio-economic position has been widely reported: the prevalence of certain specific comorbid conditions [7-10] and general comorbidity prevalence being higher in deprived groups of patients [11-13]. Our second aim was to describe patterns of comorbidities and multiple comorbidity in these cancer patient cohorts, according to patient characteristics such as socioeconomic position (deprivation).

\section{Methods}

We defined a comorbid condition as one of the following fourteen health conditions: myocardial infarction (MI), congestive heart failure (CHF), peripheral vascular disease (PVD), cerebrovascular disease (CVD), dementia, chronic obstructive pulmonary disease (COPD), rheumatological conditions, liver disease, diabetes, hemiplegia or paraplegia, renal disease, previous malignancy, obesity or hypertension. The conditions, selected following a systematic search of the data, included conditions of the Charlson Comorbidity Index [6] and any highly prevalent conditions that may influence cancer management alone or in combination with another condition.

\section{Data}

This study used England National Cancer Registry data of 331,655 patients aged 15-90 years at diagnosis with cancer of the colon, rectum, lung or Hodgkin's lymphoma, between 2009 and 2013. Registry data provided information on patient sex, age at diagnosis, site of cancer, date of cancer diagnosis and area of residence at time of diagnosis, which was used to derive socio-economic position, based on deprivation quintiles of the Income Domain of the Indices of Multiple Deprivation [14]. The five-level, ordinal variable indicates the level of deprivation from 1 (least deprived) to 5 (most deprived). Areas of residence are defined at the Lower Super Output Area level (mean population 1500).

Inpatient, outpatient and emergency hospital admissions records (Hospital Episode Statistics, HES) [15] were successfully linked with over $99 \%$ of the cancer registry records, using common unique variables present in both data sources. The International Statistical Classification of Diseases and Related Health Conditions tenth edition (ICD-10) [16] codes captured within the diagnostic fields of HES records provided information on health conditions recorded during hospital admissions. We used the ICD-10 code groupings of health conditions proposed by Quan and colleagues for defining comorbidities using administrative data (see Additional file 1) [17], and used an algorithm [18] to identify whether these conditions had been recorded in the six-year period prior to cancer diagnosis. In contrast to the approach of Maringe and colleagues [18], we included diagnoses of conditions recorded up to 6 months prior to cancer diagnosis. We anticipated that first-time diagnoses of the conditions could occur in this period, and wanted to obtain the most complete picture of patient comorbidity. We used cancer registry data to identify whether a patient had been diagnosed with an unrelated malignancy up to 6 years before their diagnosis with the cancer of interest.

\section{Descriptive data analysis}

We calculated the prevalence of a comorbid condition within each of the four patient cohorts defined by cancer site, firstly as a crude measure, calculating the percentage of patients who had a recorded diagnosis of the comorbidity in HES records, and secondly adjusting for 
age and sex to account for the older age demographic of cancer patient populations. Weights for this adjustment were obtained from 2011 UK census published population estimates of persons living in England [19].

\section{Statistical analysis}

Logistic regression models were used to estimate the odds ratio (OR) of having each comorbidity by cancer site, adjusting for sex, age at cancer diagnosis and deprivation group. The binary outcome variable indicated the presence of the comorbidity. To account for a non-linear association between increasing age and the presence of comorbidity, age was modelled as a continuous variable using a restricted cubic spline with one knot fixed at 70 years in analyses conducted for cancers of the colon, rectum and lung and at 45 years for HL (the knot position was chosen as to be close to the mean age of the patients in each of these cancer cohorts). To reduce the risk of unstable models, we ensured there were at least ten or more occurrences of a comorbidity within the specific cancer patient cohort for every parameter of the model (events per variable, EPV) [20].

Multinomial logistic regression was used to estimate the probability of having a given comorbidity, either in isolation, or as one of multiple comorbidities, according to cancer site. The three-category outcome variable indicated whether the patient did not have the given comorbidity, only had this comorbidity, or had this comorbidity with other comorbidities. Models were adjusted for age, sex and deprivation, and were run for each cancer site and comorbidity combination with at least ten EPV.

All data analyses were conducted in STATA v.15.1 [21].

\section{Results}

\section{Patient characteristics}

The characteristics of patients diagnosed with cancer of the colon $(N=102,216)$, rectum $(N=56,342)$, lung $(N=$ $165,677)$ or with HL $(N=7420)$ between 2009 and 2013, stratified by comorbidity status, are shown in Table 1 . The majority of patients in each cohort were male: approximately $55 \%$ of colon, lung and $\mathrm{HL}$ patients and $63 \%$ of rectal cancer patients. At least $80 \%$ of colon, rectum and lung cancer patients were in the two oldest age group categories, while $50 \%$ of the HL patients were within the two youngest age groups. There was an even distribution of patients among each of the deprivation groups, except among lung cancer patients, where the percentage of patients in each group increased with deprivation level.

Comorbidity was over twice as prevalent in lung cancer patients than in patients with HL: 67\% of lung cancer patients had one or more comorbidities versus almost 30\% of HL patients. Similar patterns in comorbidity prevalence were seen in males and females. The prevalence of either single or multiple comorbidity rose with increasing age. Single comorbidity was more common than multiple comorbidity in the younger age groups, whereas in the older patients the opposite was observed. For example, approximately $29.2 \%$ of lung cancer patients aged 15-29 years had one comorbidity and $3.4 \%$ had multiple comorbidities, while in lung cancer patients aged 75-90years the percentage of patients with one comorbidity or with multiple comorbidities were 26.9 and $49.9 \%$, respectively.

The prevalence of multiple comorbidity increased with deprivation level in colon, rectum and lung cancer patients, but there was no pattern with deprivation in HL patients or in the prevalence of one comorbidity. For example, from 24.7 to $25.7 \%$ of rectal cancer patients had one comorbidity, while 17.7 to $27.6 \%$ of patients had multiple comorbidities.

\section{Crude and adjusted prevalence of comorbidities at the time of cancer diagnosis}

Across all cancer patient cohorts, hypertension, COPD, diabetes, CVD, CHF and PVD were among the most commonly recorded comorbid conditions. Adjusting for age and sex strongly impacted the prevalence of some comorbid conditions in colon, rectum and lung cancer patients (Fig. 1). The three most prevalent comorbidities in all four cancer patient cohorts were hypertension, COPD and diabetes. The adjusted prevalence of hypertension and of diabetes was similar among patients in each of the four cohorts (approximately $15-20 \%$ of patients had hypertension while approximately $5 \%$ of patients had diabetes). However, the adjusted prevalence of COPD was markedly higher in patients with lung cancer: approximately $25 \%$ of lung cancer patients had COPD versus $10 \%$ of patients in the other patient cohorts. Similarly, in comparison between the four cohorts, the prevalence of several other conditions (CVD, CHF, PVD or previous malignancy) was highest among the lung cancer patients.

\section{Combinations of multiple comorbidity}

The relative frequency (\%) in which five of the most common conditions (COPD, diabetes, CVD, CHF and PVD) are present either as a single comorbidity or in combination with ten other common comorbid conditions is shown in Fig. 2. For a given cancer (identified by colour), the denominator is the number of patients with the comorbid condition, as represented on the y-axis, and the numerator is the number of those patients who had the condition as a single comorbidity or who had another condition, as depicted by the $\mathrm{x}$-axis. Patients 


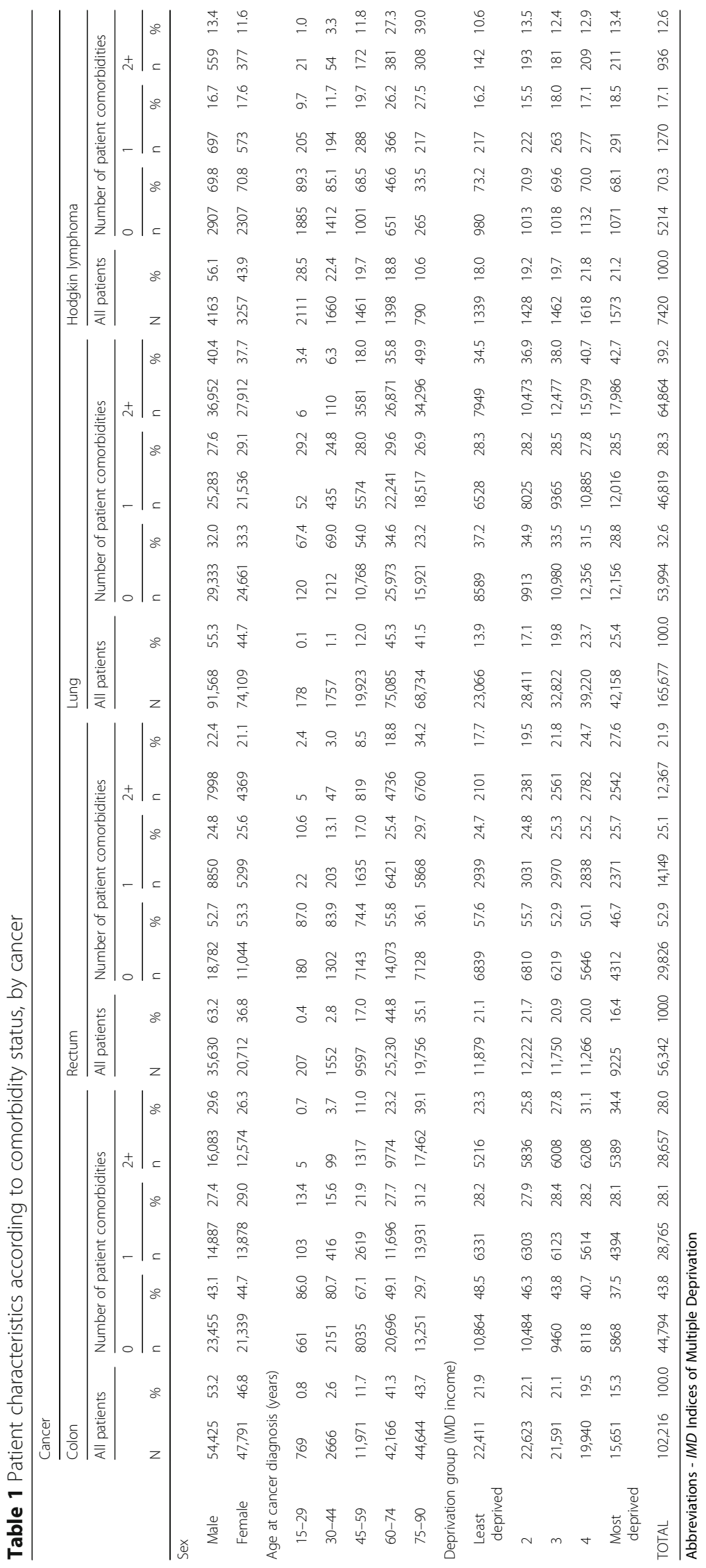




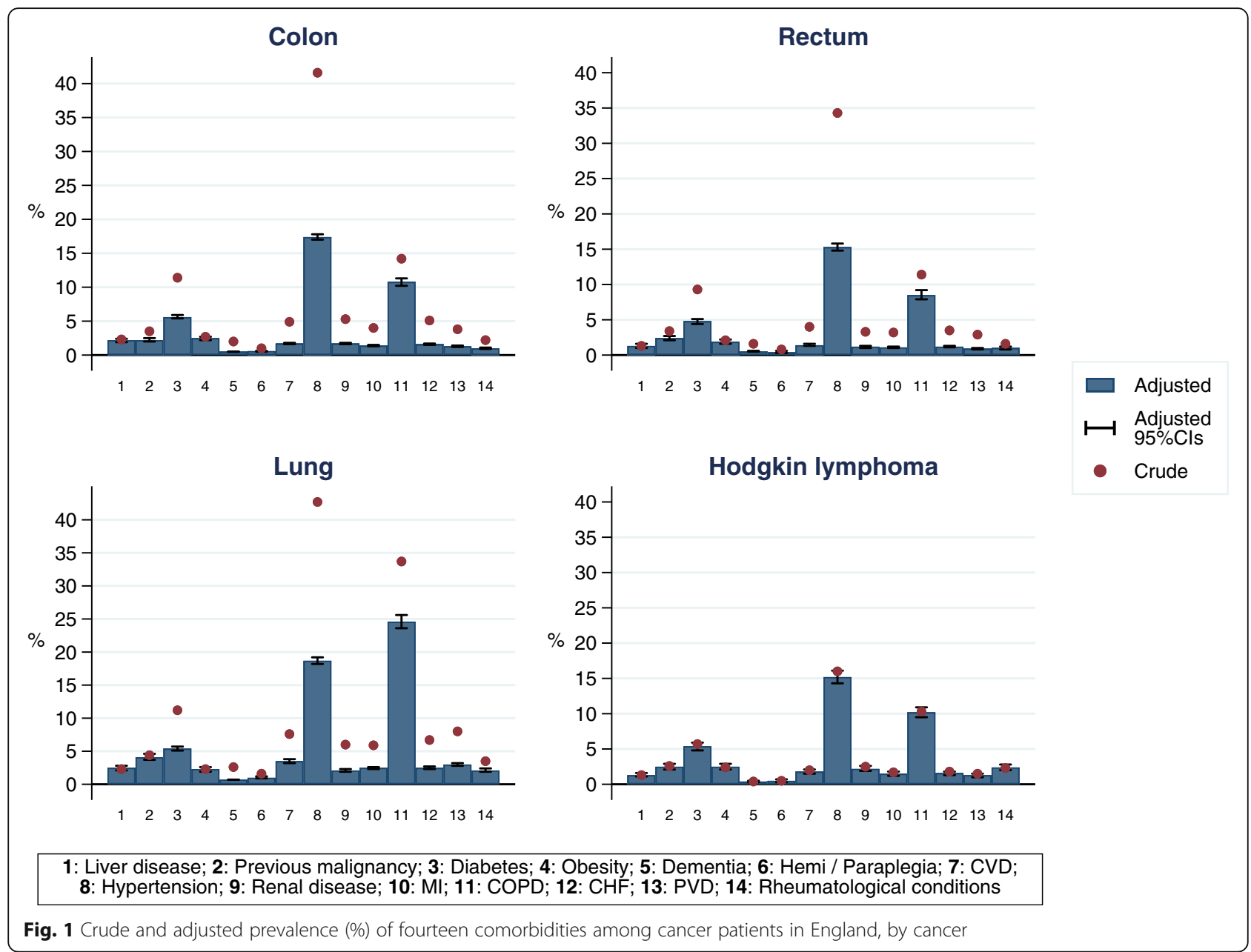

with two or more of the $\mathrm{x}$-axis conditions are represented in the numerator for each condition.

Approximately one third of colorectal and lung cancer patients with COPD, and over half of HL patients with COPD, had this condition as a single comorbidity. By comparison, under one fifth of patients with diabetes, CVD, CHF and PVD had these conditions as a single comorbidity. CHF was the condition least frequently observed as a single comorbidity across all four cancer sites (89\% or more of patients with CHF had additional comorbidities).

Hypertension was the condition most commonly present with each of comorbidities for which cross tabulations were investigated. In each of the cancer cohorts, approximately three-quarters of patients with $\mathrm{CHF}$, and a similar proportion with CVD, also had hypertension. COPD was most commonly seen in combination with diabetes, CVD, CHF or PVD in lung cancer patients: while over $50 \%$ of lung cancer patients with CHF also had COPD, around one third of patients with HL, colon or rectal cancers with CHF also had COPD.

\section{Multivariate analysis}

The odds ratios derived from logistic regression of each comorbid condition being present at the time of cancer diagnosis, by cancer site, for females relative to males, age (relative to age 70 in colon, rectal and lung cancer patients, and relative to age 45 in HL patients) and increasing deprivation, adjusted for the other listed variables, are shown in Table 2. Analyses conducted for patients with $\mathrm{HL}$ were restricted to the comorbidities of diabetes, hypertension and COPD, as the prevalence counts of the other conditions did not adhere to the minimum of ten EPV required for the analyses.

Female patients with colon, rectal or lung cancer had up to $29 \%$ increased adjusted odds of having dementia (rectal cancer: OR 1.29; 95\%CI 1.13, 1.48), up to $34 \%$ increased adjusted odds of having a previous malignancy (rectal cancer: OR $1.34 ; 1.23,1.47$ ) and approximately twice the adjusted odds of having rheumatological conditions (colon cancer: OR 2.16; 1.98, 2.36) compared to male patients. Conversely, compared with male patients in their respective cohort, females had significantly reduced adjusted odds of having diabetes, hemiplegia or 


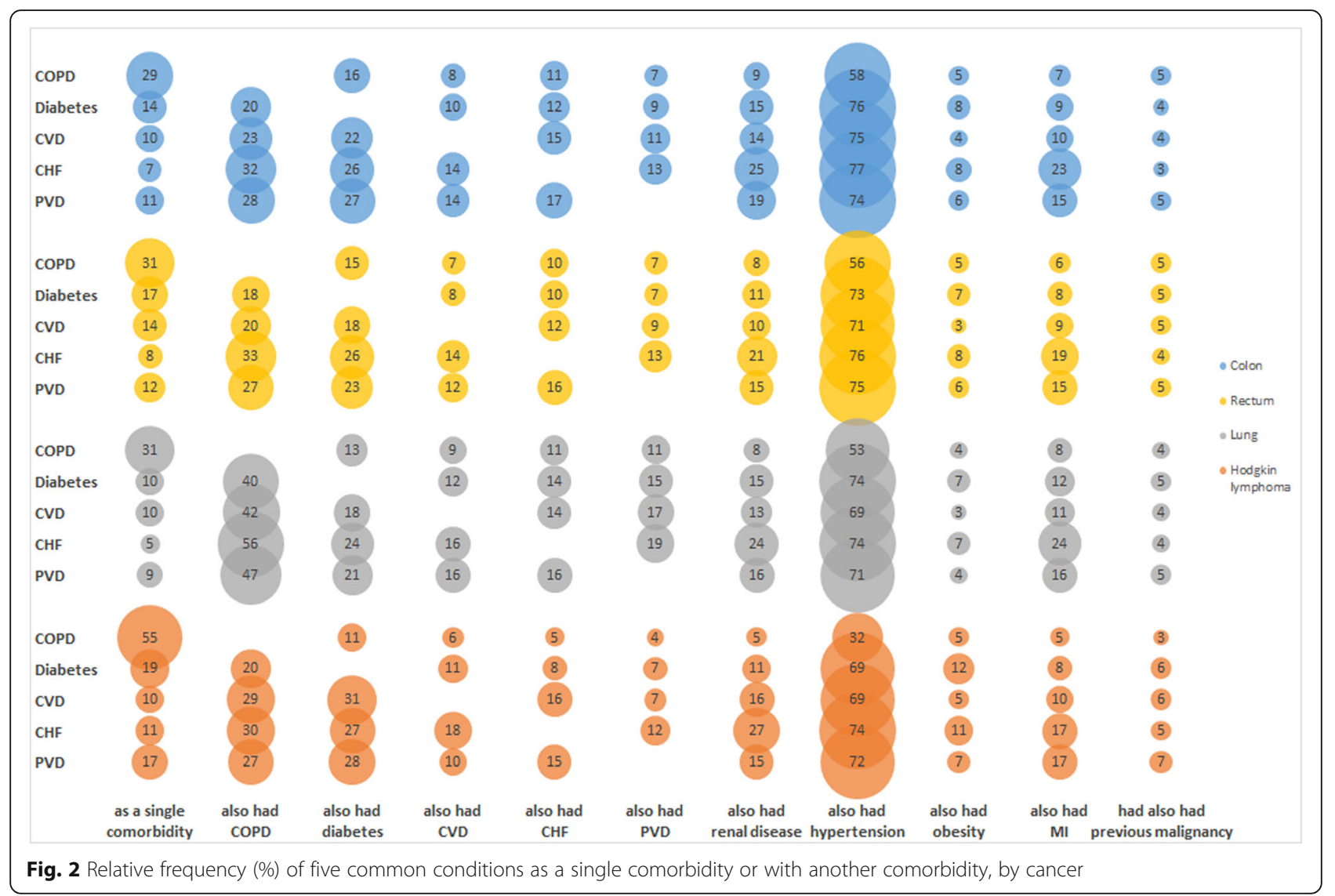

paraplegia, CVD, renal disease, MI, CHF or PVD. Across all four cancer cohorts, female patients had up to $38 \%$ reduced odds of having diabetes (HL: OR 0.62; 95\%CI $0.50,0.77$ ).

The adjusted odds of dementia, CVD, hypertension, renal disease, MI and CHF being present at diagnosis consistently increased with age. For example, with 70year old patients as the reference, colon cancer patients aged 45 had $87 \%$ reduced adjusted odds of CVD (OR $0.13 ; 0.13,0.13)$ and $88 \%$ reduced adjusted odds of CHF (OR 0.12; 0.12, 0.12), while 90-year old patients had over three times the adjusted odds of CVD (OR 3.27; 2.69, 3.99 ) and over four times the adjusted odds of CHF (OR $4.72 ; 3.63,6.13)$. There was no trend with age in colon, rectal or lung cancer patients for liver disease, having had a previous malignancy, diabetes or obesity. In lung cancer patients, no trend was observed with age for having COPD.

For at least eleven of the fourteen conditions, the adjusted odds of having the comorbid condition increased with the level of deprivation in colon, rectal or lung cancer patients. Obesity, dementia, hemiplegia, CVD, hypertension, renal disease, MI, COPD, CHF and PVD were associated with deprivation level in all three cancer cohorts. For example, the most deprived groups of lung cancer and colon cancer patients had approximately twice the adjusted odds of having COPD compared with the least deprived groups (OR 1.96; 1.89, 2.03 and OR $2.01 ; 1.89,2.12$ in the most deprived patients with lung or colon cancer, respectively). No trend with deprivation was seen with rheumatological conditions or with having a previous malignancy.

\section{Probability of having single or multiple comorbidity at the time of cancer diagnosis}

The graphs depicted in Fig. 3 show the adjusted probability of patients having one of the nine most common comorbid conditions recorded (hypertension, COPD, diabetes, CHF, CVD, PVD, MI, obesity or rheumatological conditions) at the time of colon cancer diagnosis, either as a single comorbidity, or as one of multiple comorbidities, according to age at cancer diagnosis and deprivation group (the least and most deprived groups), as derived from multinomial logistic regression.

With the exception of COPD, there was little difference between the most and least deprived groups in the probability of having each of the conditions as a single comorbidity. Among those patients with COPD as a single comorbidity, the difference in probability between 


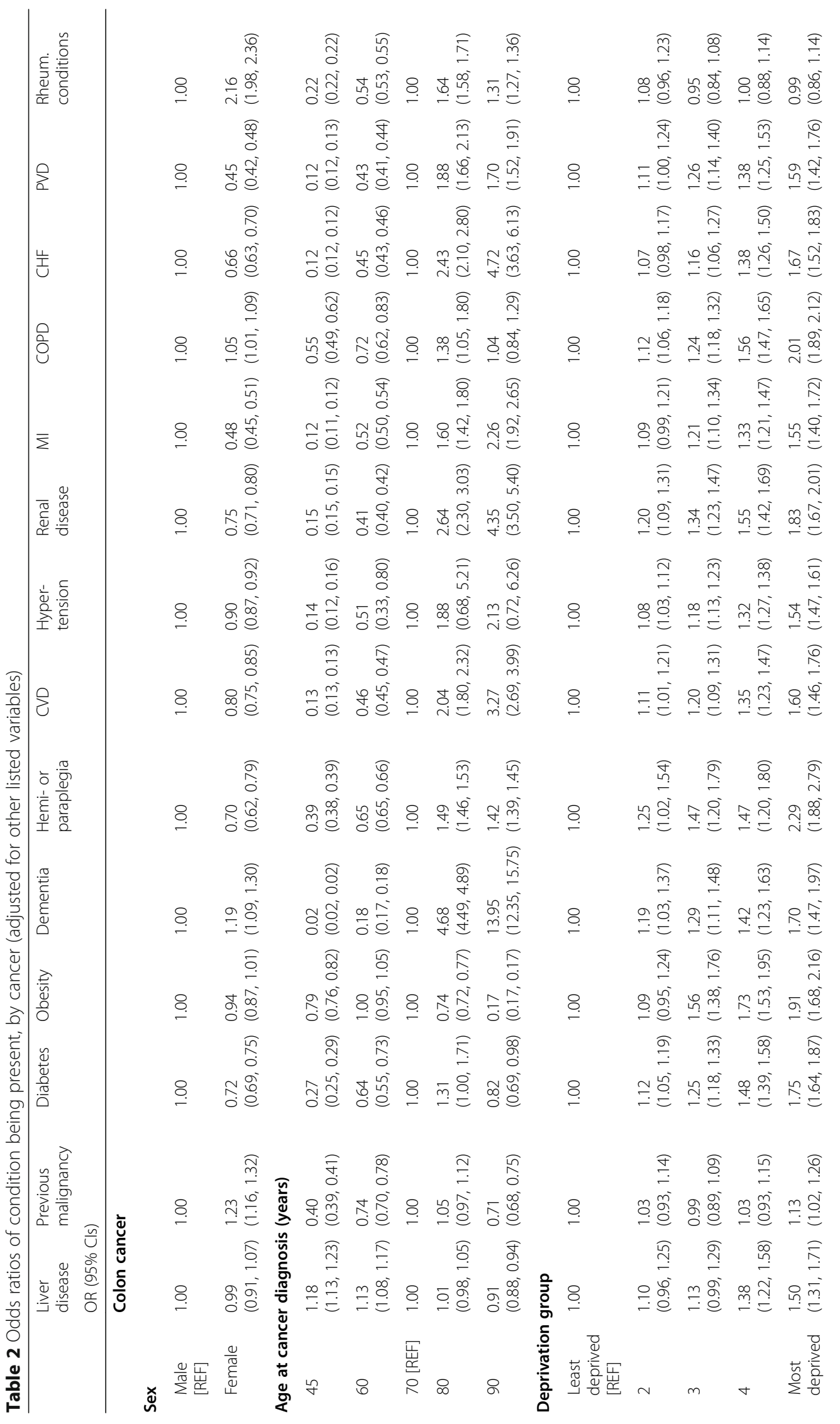




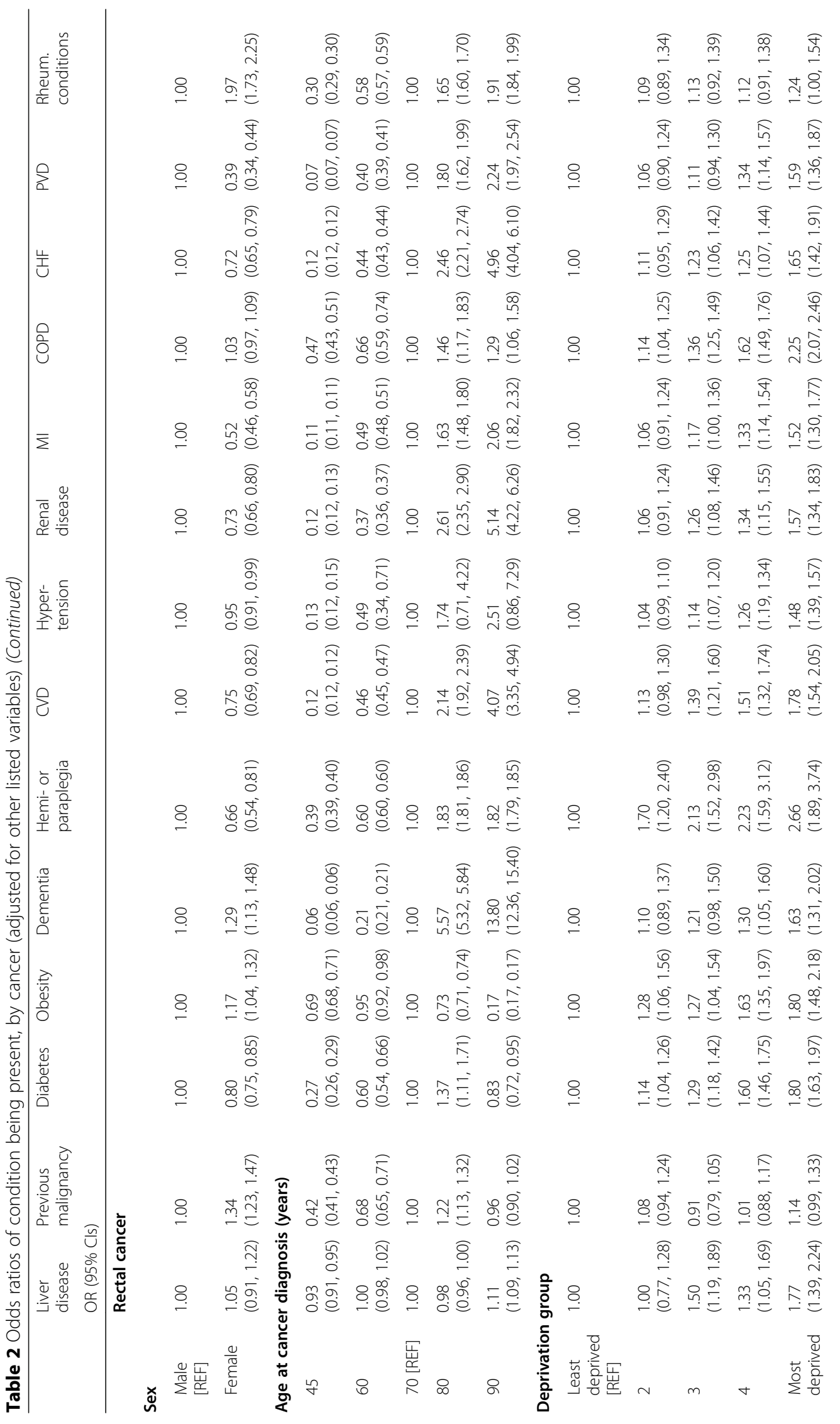




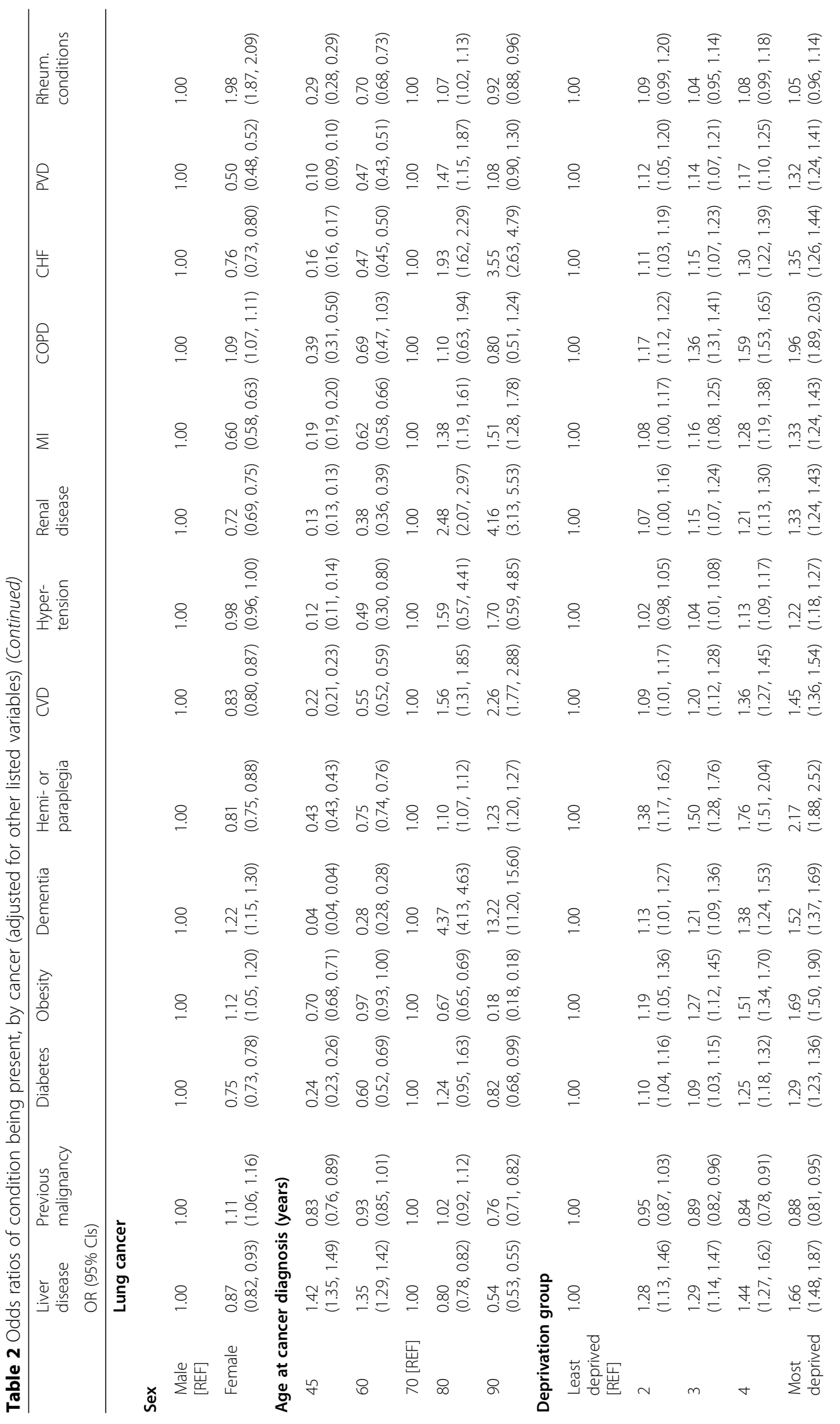


$\begin{array}{ll}\text { Fowler et al. BMC Cancer } \quad \text { (2020) 20:2 } & \end{array}$

Page 10 of 15

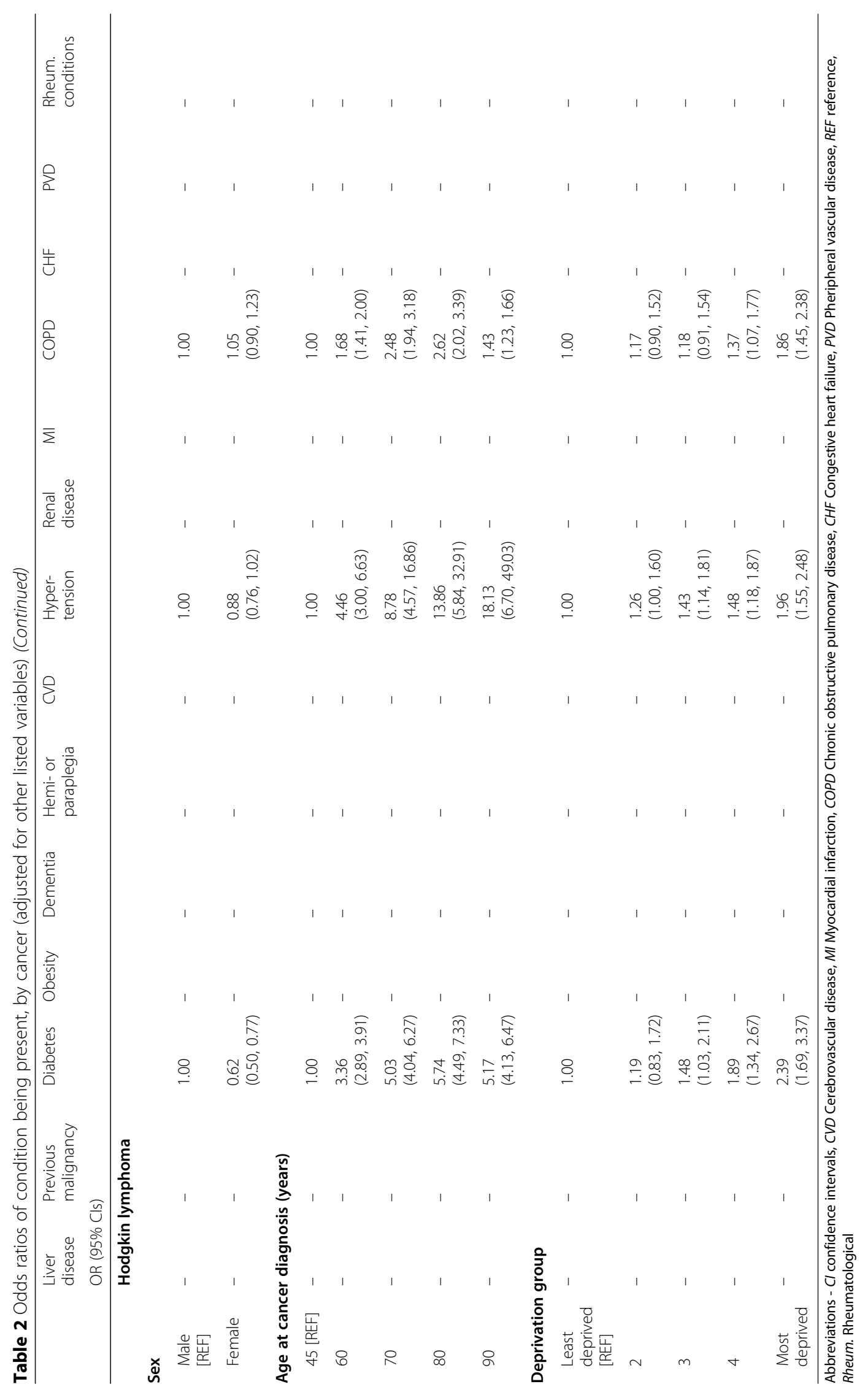




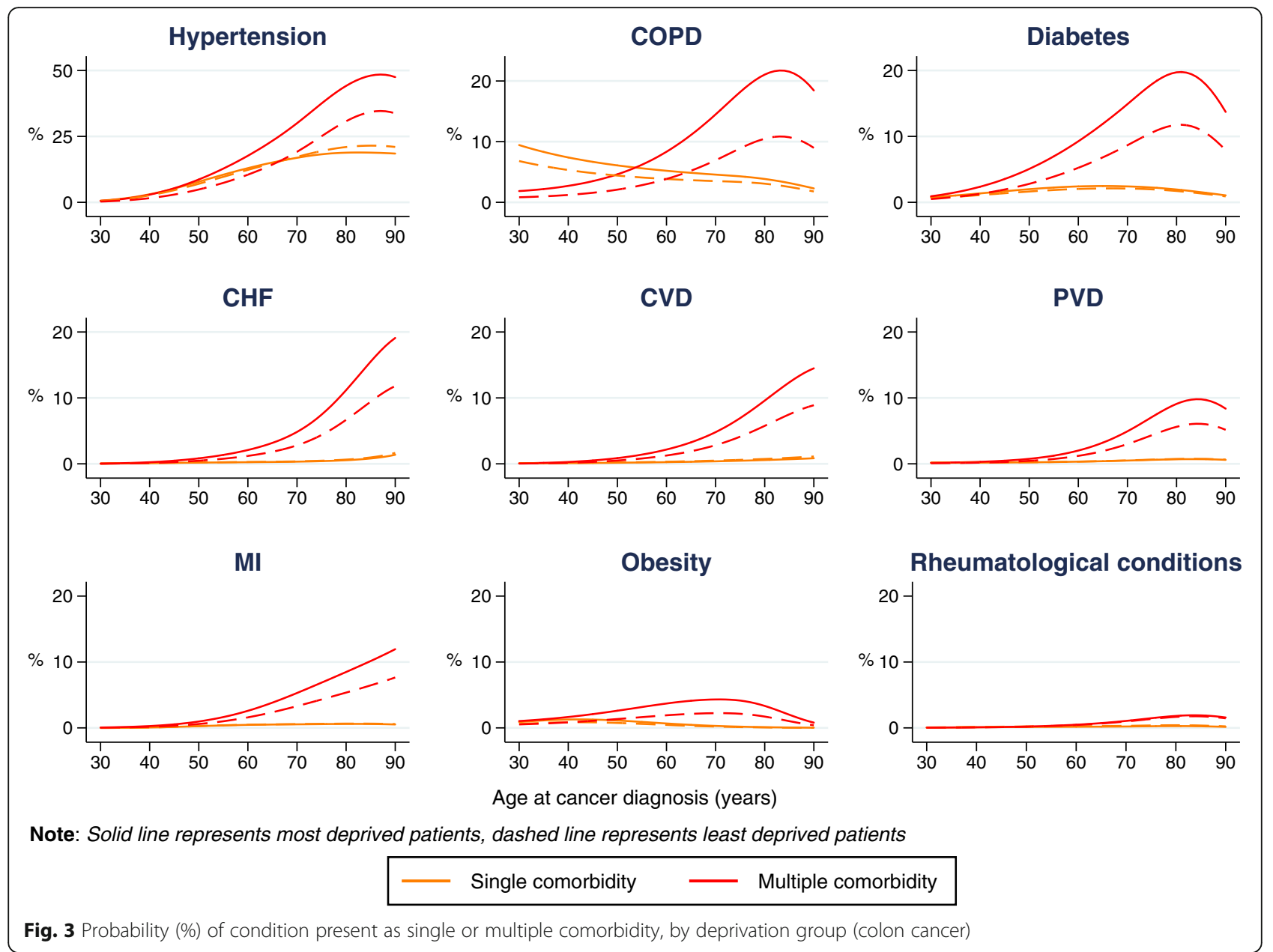

the most and least deprived groups decreased with age. The most deprived patients had a higher probability of having each of the conditions as one of multiple comorbidities compared with the least deprived group, with one exception (rheumatological conditions). Generally, the difference in probability between the two deprivation groups was greatest in older age: it peaked at approximately 80 years for hypertension, COPD, diabetes, PVD and obesity, while in patients with CHF, CVD, and MI the difference continued to increase with age. Having rheumatological conditions was not associated with increasing age or deprivation level.

Similar patterns in the probability of having a comorbid condition according to deprivation group were observed for patients with rectal or lung cancers (Additional files 2 and 3).

\section{Discussion}

Our study is, to our knowledge, the first large-scale, population-based study describing comorbidity prevalence in cancer patient populations. Up to two-thirds of patients had at least one long-term health condition at the time of their cancer diagnosis, and around half of these comorbid cancer patients had multiple longterm conditions. There was evidence that many of the comorbid conditions we investigated were associated with socio-economic deprivation, and the most deprived groups of patients had a higher probability of having multiple comorbidities compared with the less deprived groups.

The choice of cancer sites we studied was based on aetiology of the cancer: three of the cancer sites (colon, rectum and lung) were associated with environmental risk factors including tobacco smoking [22, 23], alcohol use and diet $[24,25]$. Furthermore, tobacco smoking is associated with certain conditions, such as COPD [26-28] and Type 2 diabetes [29,30], and is also associated with socioeconomic position [31]. HL is linked to infection rather than environmental factors [22].

Hypertension, COPD and diabetes were the three most prevalent comorbidities in all four cancer patient cohorts, with a higher prevalence in the most deprived patients. The odds of having COPD from being in the 
most deprived group of lung cancer patients (compared with being in the least deprived group - the 'deprivation gap') was $10 \%$ more than the deprivation gap in the adjusted odds of having COPD in the Hodgkin lymphoma patients. This may be reflective of the role of smoking in the aetiology of both lung cancer and COPD, and the higher prevalence of smoking in the more deprived population. The association between smoking status and deprivation is not quantifiable in the cancer patient cohorts as we did not have information on smoking prevalence.

Similar work using administrative data to describe comorbidity in cancer populations has been undertaken in New Zealand [32] and in Spain [33]. In the study of patients diagnosed with colon, rectal, breast, ovarian, uterine, stomach, liver, renal or bladder cancers in New Zealand $(N=14,096)$, commonly diagnosed comorbidities among colon and rectal cancer patients were hypertension, cardiac conditions and diabetes. In the Spanish cohort of colorectal cancer patients from the cancer registries of Girona and Granada $(N=1061)$, diabetes, COPD and CHF were the most common comorbidities. Comparing our study with the study in New Zealand, there were similarities among colon cancer patients in the age-sex adjusted prevalence of hypertension, while diabetes prevalence was higher in New Zealand. The adjusted prevalence of hypertension was $16.6 \%$, uncomplicated diabetes was $5.9 \%$ and diabetes with complications was $5.0 \%$ among patients in New Zealand, while in our study the adjusted prevalence of hypertension was $17.4 \%$ and diabetes (with and without complications) was $5.7 \%$. This supports our earlier assumption that less severe diabetes may be underreported in hospital admissions records. Given the 'gatekeeper' structure and functioning of the healthcare system in the UK [34] and the focus on managing diabetes within primary care [35], cases of diabetes recorded in hospital admissions are possibly those that are not controlled within available primary care resources [36] or present with complications. The Spanish study reported the crude prevalence of conditions among colorectal cancer patients, which were generally higher than the crude prevalence of conditions observed in our study. Diabetes was prevalent in $23.6 \%$ of colorectal cancer patients in this study, while in our study the crude prevalence of diabetes was $11.4 \%$ or $9.4 \%$ among colon or rectal cancer patients, respectively. Nonetheless, there was consistency between our study and both of these other studies in terms of common comorbid conditions among the patient cohorts.

In our study, approximately $13 \%$ of the HL cohort, over $21 \%$ of the colorectal cancer cohorts and over 39\% of the lung cancer cohort had multiple comorbidities, while from 17 to $28 \%$ of patients in each cohort had a single comorbidity at the time of their cancer diagnosis. These findings are important given the impact comorbidity may have on cancer care, particularly where care is provided within the constraints of healthcare guidelines that are not designed for the simultaneous management of two or more chronic conditions or morbidities (i.e. "multimorbidity"). Scientific studies indicate that multimorbidity is regularly observed in the population [37-39] and poses a challenge to health care systems, particularly those geared towards single disease management [5, 40, 41]. Clinical guidelines in the United Kingdom are not accommodating to the cumulative impact of treatment recommendations on those with multiple morbidities, and do not facilitate a comparison of potential benefits or risks [42]. Patients with multiple chronic conditions have higher rates of healthcare consultations than those without [38, 43, 44]. Managing and treating comorbid conditions places an additional economic burden on healthcare systems. In one study of the costs per capita of several comorbid conditions, renal disease was identified as one of the most costly conditions to manage among cancer patients (approximately $174 \%$ of the costs of the cancer), while the cost of diabetes or heart disease was substantially lower (approximately $20 \%$ or $6 \%$ of cancer costs, respectively) [45]. The increase in costs also depends on the number and combination of comorbid conditions: among the cancer patients with diabetes in our study, between 10 and $15 \%$ of these patients also had renal disease.

In cancer patients, the presence of comorbidity can be influential on cancer management and therapeutic options. Patients with comorbidity may be less likely than those without comorbidity to receive curative treatment [3]. Treatment decisions made by clinicians may be weighted by the type and severity of comorbidity, for example, CHF has been reported to influence receipt of surgery for non-small cell lung cancer [46], receipt of adjuvant chemotherapy for colon cancer [47] and receipt of any treatment for prostate cancer [48]. The presence of COPD influenced receipt of surgical treatment in non-small cell lung cancer patients [46] and adjuvant therapy in colon cancer patients [47]. However, there is also evidence that comorbid patients who receive treatment have better prognosis for survival than those who do not receive treatment, as shown with the receipt of adjuvant therapy for colon cancer [47, 49]. Moreover, older cancer patients and patients with comorbidity have historically been under-represented in cancer clinical trials. This limits the applicability of cancer clinical trial results to a younger and healthier cohort of patients than clinicians are actually treating, meaning that while there is evidence suggesting that patients with comorbidity as a group are not receiving optimal cancer treatment, specific information required for clinical decisionmaking is often lacking [50]. We found a non-negligible increase in the prevalence of comorbidities when we 
included diagnoses in the six-months prior to cancer diagnoses. While some of these conditions may have arisen in these months because of the cancer, their presence will be as relevant when considering treatment, irrespective of the timing of their diagnosis.

Our study showed socio-economic position to be an important factor associated with having one or more comorbid conditions at the time of cancer diagnosis, with comorbidity prevalence increasing with deprivation. It is possible that mechanisms within clinical guidelines and decision-making that lead to non-treatment of cancer patients with comorbidity disproportionately impact the more deprived patients. An existence of socio-economic inequalities in receipt of treatment has been identified $[51,52]$. Reviewing the treatment process of cancer patients with comorbidity may therefore have a beneficial effect in reducing the socioeconomic inequalities in receipt of cancer treatment. Moreover, because cancer data contains mainly cancer-related outcomes, how the cancer and related treatments impact patient comorbidity and prognosis is not well known [3]. Having the resources and guidelines within which to manage patient comorbid conditions robustly during cancer treatment is one strategy for mitigating the risk of adverse patient outcomes occurring from comorbid disease. In England, socio-economic inequalities in cancer survival have narrowed little, despite the implementation of government strategies that intended to reduce these inequalities [53]. Focusing on the management of comorbidity in cancer patients could be one potential pathway to addressing socio-economic inequalities in cancer outcomes.

There are a variety of metrics of comorbidity in the scientific literature that are used to study the relationship between comorbidity on cancer outcomes, although no consensus has been reached on a gold standard measure of comorbidity within the context of cancer [54]. Many of the approaches provide a summary measure of the patient's comorbid conditions and the severity of these conditions. However, the prognostic impact of comorbidity can depend on the type and stage of the cancer [55]. In addition, the presence of comorbidity particularly certain comorbid conditions - adds complexity to the provision of treatment for cancer. When investigating the relationship between comorbidity and cancer outcomes, a more granular approach investigating specific comorbid conditions in turn, rather than using a summary measure of comorbidity, could be more appropriate and insightful.

We acknowledge potential limitations in this study. We capture comorbidity information based on diagnoses of health conditions recorded during hospital admission(s) prior to cancer diagnosis, and are therefore reliant on patients requiring hospital-based medical attention for their health condition(s) in order to obtain this information. The potential for measurement error from the information recorded in the diagnostic fields of hospital admissions records should also be acknowledged. However, we assume that the more severe conditions are likely to be captured within the diagnostic fields. Underreporting may occur in less severe conditions, such as obesity, that are unlikely to be the primary reason for the hospital admission, and may occur more frequently with elderly patients or patients with more severe comorbidities, due to competing demands. Conditions such as less severe type II diabetes are possibly underreported. Further work comparing the prevalence of the conditions we studied in the cancer cohorts with the prevalence of these conditions in the general population in England, as reported in government publications and scientific literature, would be useful step in validating our results.

Our study of over 300,000 patients is one of the largest population-based studies of comorbidity prevalence among cancer patients, and one of the first such studies of patients in England. Using data from well-established sources, we were able to describe the prevalence of fourteen chronic health conditions among these cancer patients, and highlight an association between socioeconomic position and prevalence of most of these conditions.

\section{Conclusion}

This study underlines that many comorbid cancer patients are living with multiple comorbidities, and that the most deprived patients carry the greater burden of comorbidity. Healthcare guidelines may not always encompass the simultaneous management of multiple chronic conditions, but guidelines for the management of cancer may need to consider some prominent comorbid conditions. Insight into patterns of cancer comorbidity informs further research into the influence of comorbidity - particularly the influence of specific comorbid conditions - on outcomes following cancer diagnosis, including socio-economic inequalities in receipt of treatment and short-term mortality.

\section{Supplementary information}

Supplementary information accompanies this paper at https://doi.org/10. 1186/s12885-019-6472-9.

Additional file 1. Definition of the fourteen conditions, according to ICD-10 code classification. Table of the fourteen conditions and the ICD10 code groupings used to define them.

Additional file 2. Probability (\%) of condition present as single or multiple comorbidity, by deprivation group (lung cancer). Additional results in complement to those presented in Fig. 3: graphs representing the probability of having any of nine comorbid conditions in lung cancer patients. 
Additional file 3. Probability (\%) of condition present as single or multiple comorbidity, by deprivation group (rectal cancer). Additional results in complement to those presented in Fig. 3: graphs representing the probability of having any of nine comorbid conditions in rectal cancer patients.

\section{Abbreviations}

CCl: Charlson Comorbidity Index; CHF: Congestive Heart Failure; $\mathrm{Cl}$ : Confidence Interval; COPD: Chronic Obstructive Pulmonary Disease; CVD: Cerebrovascular Disease; EPV: Events Per Variable; HES: Hospital Episode Statistics; HL: Hodgkin lymphoma; ICD: International Classification of Diseases and Related Health Problems; Ml: Myocardial infarction; OR: Odds Ratio; PVD: Peripheral Vascular Disease

\section{Acknowledgements}

We would like to thank Adrian Turculet, Data Manager of the LSHTM Cancer Survival Group, for his support and assistance with the data linkage.

\section{Authors' contributions}

$C M, M A L F$ and HF contributed to the conception of the study. BR, HF, AB and LE designed the study. AB and ENN provided advice on statistical methods. HF conducted the analyses of the data and prepared the draft of the manuscript, tables and figures. BR, $A B$, and $L E$ supervised the study and provided comments on the manuscript draft. CM, MALF, NN and DS provided comments on the final draft of the manuscript. All authors read and approved the final manuscript.

\section{Funding}

This research was funded by Cancer Research UK grant number C7923/ A18525. The authors declare no support from any organisations for the submitted work. The design of the study, the analyses and the writing of the manuscript were solely the responsibility of the authors. The findings and conclusions in this manuscript are those of the authors and do not necessarily represent the views of Cancer Research UK.

\section{Availability of data and materials}

The data that support the findings of this study are available via application to the Public Health England Office for Data Release, but restrictions apply to the availability of these data.

\section{Ethics approval and consent to participate}

We obtained the statutory approvals required for this research from the Confidentiality Advisory Group (CAG) of the Health Research Authority (HRA): PIAG 1-05(c) 2007

Ethical approval was obtained from the Research Ethics Committee (REC) of the Health Research Authority (HRA): 07/MRE01/52.

This work uses data provided by patients and collected by the National Health Service as part of their care and support. We used anonymised National Cancer Registry and Hospital Episode Statistics data. No consent to participate was sought from patients.

\section{Consent for publication}

Not applicable.

\section{Competing interests}

The authors declare that they have no competing interests.

\section{Author details}

${ }^{1}$ Cancer Survival Group, Department of Non-Communicable Disease Epidemiology, London School of Hygiene \& Tropical Medicine, Keppel Street, London WC1E 7HT, UK. ${ }^{2}$ Biomedical Research Institute of Granada, Non-Communicable and Cancer Epidemiology Group, University of Granada, Granada, Spain. ${ }^{3}$ Department of Non-Communicable Disease Epidemiology, London School of Hygiene \& Tropical Medicine, London, UK. ${ }^{4} \mathrm{UCL}$ Respiratory, University College London, London, UK. ${ }^{5}$ Department of Thoracic Medicine, University College London Hospital, London, UK. ${ }^{6}$ Department of Public Health, University of Otago, Wellington, New Zealand.
Received: 8 May 2019 Accepted: 17 December 2019

Published online: 28 January 2020

\section{References}

1. Porta MS, Greenland S, Last JM. A dictionary of epidemiology: Oxford University press; 2014. Available from: http://www.oxfordreference.com/ view/10.1093/acref/9780195314496.001.0001/acref-9780195314496-e-352 ?rskey=TmAWeL\&result=351. Accessed 31 Oct 2018.

2. Gumey J, Sarfati D, Stanley J. The impact of patient comorbidity on cancer stage at diagnosis. BJC. 2015;113:1375-80

3. Sarfati D, Koczwara B, Jackson C. The impact of comorbidity on cancer and its treatment. CA Cancer J Clin. 2016;66(4):337-50.

4. Salika T, Lyratzopoulos G, Whitaker KL, Waller J, Renzi C. Do comorbidities influence help-seeking for cancer alarm symptoms? A population-based survey in England. J Public Health. 2018;40(2):340-9.

5. Tinetti ME, Fried TR, Boyd CM. Designing health care for the most common chronic condition--multimorbidity. JAMA. 2012;307(23):2493-4.

6. Charlson ME, Pompei P, Ales KL, Mackenzie CR. A new method of classifying prognostic comorbidity in longitudinal studies: development and validation. J Chronic Dis. 1987;40(5):373-83.

7. Connolly V, Unwin N, Sherriff P, Bilous R, Kelly W. Diabetes prevalence and socioeconomic status: a population based study showing increased prevalence of type 2 diabetes mellitus in deprived areas. J Epidemiol Community Health. 2000:54(3):173-7.

8. Sundquist K, Malmstrom M, Johansson S. Neighbourhood deprivation and incidence of coronary heart disease: a multilevel study of 2.6 million women and men in Sweden. J Epidemiol Community Health. 2004;58(1):71-7.

9. Hossain MP, Palmer D, Goyder E, El Nahas AM. Social deprivation and prevalence of chronic kidney disease in the UK: workload implications for primary care. QJM. 2012;105(2):167-75.

10. Fano V, Pezzotti P, Gnavi R, Bontempi K, Miceli M, Pagnozzi E, et al. The role of socio-economic factors on prevalence and health outcomes of persons with diabetes in Rome, Italy. Eur J Public Health. 2013;23(6):991-7.

11. Macleod U, Mitchell E, Black M, Spence G. Comorbidity and socioeconomic deprivation: an observational study of the prevalence of comorbidity in general practice. Eur J Gen Pract. 2009;10(1):3.

12. McLean G, Gunn J, Wyke S, Guthrie B, Watt G, Blane D, et al. The influence of socioeconomic deprivation on multimorbidity at different ages: a crosssectional study. Br J Gen Pract. 2014;64(624):8.

13. Moffat K, Mercer SW. Challenges of managing people with multimorbidity in today's healthcare systems. BMC Fam Pract. 2015;16:129.

14. Department for Communities and Local Government. The English Indices of Deprivation 2010. London: 2011. Available from: https://www.gov.uk/ government/statistics/english-indices-of-deprivation-2010

15. Health and Social Care Information Centre. Hospital Episode Statistics 2013. Available from: http://www.hscic.gov.uk/hes. Accessed 18 Nov 2017.

16. World Health Organisation. International statistical classification of diseases and related health problems. Tenth revision. Geneva: World Health Organization; 1994

17. Quan HD, Li B, Couris CM, Fushimi K, Graham P, Hider P, et al. Updating and validating the Charlson comorbidity index and score for risk adjustment in hospital discharge abstracts using data from 6 countries. Am J Epidemiol. 2011;173(6):676-82.

18. Maringe C, Fowler H, Rachet B, Luque-Fernández MA. Reproducibility, reliability and validity of population-based administrative health data for the assessment of cancer non-related comorbidities. PLoS One. 2017:12(3):e0172814

19. Office for National Statistics. 2011 Census: Population estimates by single year of age and sex for local authorities in the United Kingdom 2013. Available from: https://www.ons.gov.uk/peoplepopulationandcommunity/ populationandmigration/populationestimates/datasets/2011 censuspopulationestimatesbysingleyearofageand sexforlocalauthoritiesintheunitedkingdom. Accessed 01 Aug 2018.

20. Peduzzi P, Concato J, Kemper E, Holford TR, Feinstein AR. A simulation study of the number of events per variable in logistic regression analysis. J Clin Epidemiol. 1996;49(12):1373-9.

21. StataCorp. STATA statistical software. 15th ed. College Station: Stata Corporation; 2017.

22. Schottenfeld D, Fraumeni JF. Cancer epidemiology and prevention. 3rd ed. Oxford: Oxford University Press; 2006.

23. Tindle HA, Stevenson Duncan M, Greevy RA, Vasan RS, Kundu S, Massion PP, et al. Lifetime Smoking History and Risk of Lung Cancer: Results From the Framingham Heart Study. J Natl Cancer Inst. 2018;110(11):1201-7. 
24. Danaei G, Vander Hoorn S, Lopez AD, Murray CJ, Ezzati M. Causes of cancer in the world: comparative risk assessment of nine behavioural and environmental risk factors. Lancet. 2005;366(9499):1784-93.

25. Haggar FA, Boushey RP. Colorectal cancer epidemiology: incidence, mortality, survival, and risk factors. Clin Colon Rectal Surg. 2009;22(4):191-7.

26. Devereux G. ABC of chronic obstructive pulmonary disease - definition, epidemiology, and risk factors. BMJ. 2006;332(7550):1142-4.

27. Buist AS, McBurnie MA, Vollmer WM, Gillespie S, Burney P, Mannino DM, et al. International variation in the prevalence of COPD (the BOLD study): a population-based prevalence study. Lancet. 2007;370(9589):741-50.

28. Laniado-Laborín R. Smoking and chronic obstructive pulmonary disease (COPD). Parallel epidemics of the 21(st) century. Int J Environ Res Public Health. 2009;6(1):209-24.

29. Hu FB, Manson JE, Stampfer MJ, Colditz G, Liu S, Solomon CG, et al. Diet, lifestyle, and the risk of type 2 diabetes mellitus in women. $\mathrm{N}$ Engl J Med. 2001;345(11):790-7.

30. Wannamethee SG, Shaper AG, Perry IJ. Smoking as a modifiable risk factor for type 2 diabetes in middle-aged men. Diabetes Care. 2001;24(9):1590-5.

31. Hiscock R, Bauld L, Amos A, Platt S. Smoking and socioeconomic status in England: the rise of the never smoker and the disadvantaged smoker. J Public Health. 2012;34(3):390-6

32. Sarfati D, Gurney J, Lim BT, Bagheri N, Simpson A, Koea J, et al. Identifying important comorbidity among cancer populations using administrative data: prevalence and impact on survival. Asia Pac J Clin Oncol. 2016;12(1):e47-56.

33. Luque-Fernandez MA, Redondo Sánchez D, Rodriguez-Barranco M, CarmonaGarcia MC, Marcos-Gragera R, Sanchez-Perez MJ. Multimorbidity by patient and tumor factors and time-to-surgery among colorectal cancer patients in Spain: a population-based study. Clinical Epidemiology. 2019 [in press].

34. Loudon I. The principle of referral: the gatekeeping role of the GP. Br J Gen Pract. 2008:58(547):128-30 discussion 30.

35. Nicholson E, Cummings M, Cranston I, Meeking D, Kar P. The super six model of care: five years on. Diab Prim Care. 2016;18:6.

36. Saxena S, George J, Barber J, Fitzpatrick J, Majeed A. Association of population and practice factors with potentially avoidable admission rates for chronic diseases in London: cross sectional analysis. J R Soc Med. 2006; 99(2):81-9.

37. Fortin M, Bravo G, Hudon C, Vanasse A, Lapointe L. Prevalence of multimorbidity among adults seen in family practice. Ann Fam Med. 2005; 3(3):223-8.

38. Salisbury C, Johnson L, Purdy S, Valderas JM, Montgomery AA. Epidemiology and impact of multimorbidity in primary care: a retrospective cohort study. Br J Gen Pract. 2011;61(582):e12-21.

39. Schäfer I, Hansen H, Schön G, Höfels S, Altiner A, Dahlhaus A, et al. The influence of age, gender and socio-economic status on multimorbidity patterns in primary care. first results from the multicare cohort study. BMC Health Serv Res. 2012;12(1):89.

40. Boyd CM, Fortin M. Future of multimorbidity research: how should understanding of multimorbidity inform health system design? Public Health Rev. 2010;32(2):451-74.

41. Barnett K, Mercer SW, Norbury M, Watt G, Wyke S, Guthrie B. Epidemiology of multimorbidity and implications for health care, research, and medical education: a cross-sectional study. Lancet. 2012;380(9836):37-43.

42. Hughes LD, McMurdo MET, Guthrie B. Guidelines for people not for diseases: the challenges of applying UK clinical guidelines to people with multimorbidity. Age Ageing. 2013;42(1):62-9.

43. Glynn LG, Valderas JM, Healy P, Burke E, Newell J, Gillespie P, et al. The prevalence of multimorbidity in primary care and its effect on health care utilization and cost. Fam Pract. 2011;28(5):516-23.

44. Bahler C, Huber CA, Brungger B, Reich O. Multimorbidity, health care utilization and costs in an elderly community-dwelling population: a claims data based observational study. BMC Health Serv Res. 2015;15:23.

45. Cortaredona S, Ventelou B. The extra cost of comorbidity: multiple illnesses and the economic burden of non-communicable diseases. BMC Med. 2017;15(1):216.

46. Belot A, Fowler H, Njagi EN, Luque-Fernandez MA, Maringe C, Magadi W, et al. Association between age, deprivation and specific comorbid conditions and the receipt of major surgery in patients with non-small cell lung cancer in England: a population-based study. Thorax. 2019;74(1):51-9.

47. Gross CP, McAvay GJ, Guo Z, Tinetti ME. The impact of chronic illnesses on the use and effectiveness of adjuvant chemotherapy for colon cancer. Cancer. 2007:109(12):2410-9.
48. Bradley CJ, Dahman B, Anscher M. Prostate cancer treatment and survival: evidence for men with prevalent comorbid conditions. Med Care. 2014; 52(6):482-9.

49. Sarfati D, Hill S, Blakely T, Robson B, Purdie G, Dennett E, et al. The effect of comorbidity on the use of adjuvant chemotherapy and survival from colon cancer: a retrospective cohort study. BMC Cancer. 2009;9:116.

50. Aapro MS, Kohne $\mathrm{CH}$, Cohen HJ, Extermann M. Never too old? Age should not be a barrier to enrollment in cancer clinical trials. Oncologist. 2005;10(3): 198-204.

51. Akinyemiju T, Meng Q, Vin-Raviv N. Race/ethnicity and socio-economic differences in colorectal cancer surgery outcomes: analysis of the nationwide inpatient sample. BMC Cancer. 2016;16:715.

52. Forrest LF, Sowden S, Rubin G, White M, Adams J. Socio-economic inequalities in stage at diagnosis, and in time intervals on the lung cancer pathway from first symptom to treatment: systematic review and metaanalysis. Thorax. 2017;72(5):430-6.

53. Exarchakou A, Rachet B, Belot A, Maringe C, Coleman MP. Impact of national cancer policies on cancer survival trends and socioeconomic inequalities in England, 1996-2013: population based study. BMJ. 2018;360:k764.

54. Sarfati D. Review of methods used to measure comorbidity in cancer populations: no gold standard exists. J Clin Epidemiol. 2012;65(9):924-33.

55. Read WL, Tierney RM, Page NC, Costas I, Govindan R, Spitznagel EL, et al. Differential prognostic impact of comorbidity. J Clin Oncol. 2004;22(15): 3099-103.

\section{Publisher's Note}

Springer Nature remains neutral with regard to jurisdictional claims in published maps and institutional affiliations.

Ready to submit your research? Choose BMC and benefit from:

- fast, convenient online submission

- thorough peer review by experienced researchers in your field

- rapid publication on acceptance

- support for research data, including large and complex data types

- gold Open Access which fosters wider collaboration and increased citations

- maximum visibility for your research: over $100 \mathrm{M}$ website views per year

At $\mathrm{BMC}$, research is always in progress.

Learn more biomedcentral.com/submissions 